\title{
Perfil epidemiológico do suicídio no extremo oeste do estado de Santa Catarina, Brasil
}

\author{
Epidemiological profile of suicide in the west of the State of Santa Catarina, Brazil
}

\author{
Ricardo Schmitt ${ }^{1}$, Maria Gabriela Lang ${ }^{2}$, João Quevedo ${ }^{3}$, Talita Colombo ${ }^{2}$ \\ 1 Mestre. Professor titular, Centro de Ciências da Saúde, Universidade Comunitária Regional de Chapecó (UNOCHAPECÓ), Chapecó, SC. \\ Coordenador, Grupo de Pesquisa em Epidemiologia Clínica (EPICLIN), Chapecó, SC. ${ }^{2}$ Acadêmica de Medicina, UNOCHAPECÓ. Pesquisadora, \\ EPICLIN. ${ }^{3}$ Doutor em Ciências Biológicas: Bioquímica. Professor titular e coordenador, Programa de Pós-Graduação em Ciências da Saúde, \\ Unidade Acadêmica de Ciências da Saúde, Universidade do Extremo Sul Catarinense (UNESC), Criciúma, SC.
}

\section{Resumo}

Introdução: O estado de Santa Catarina está localizado na Região Sul do Brasil. O coeficiente de mortalidade nesse estado é um dos maiores do país. Estudos preliminares indicam que a região do extremo oeste de Santa Catarina apresenta os maiores índices estaduais. Inexistem estudos descritivos sobre o perfil epidemiológico do suicídio nessa região.

Método: Estudo descritivo, calculando o coeficiente bruto de mortalidade por suicídio no extremo oeste catarinense, assim como os coeficientes padronizados por gênero e faixa etária no período entre 1980 e 2005 . Descrição dos meios empregados para o suicídio. Os dados foram coletados do Departamento de Informática do Sistema Único de Saúde.

Resultados: O coeficiente médio de suicídios no extremo oeste foi de 10 casos para cada 100.000 habitantes. A proporção entre homens e mulheres foi de 3:1. Os índices apresentaram aumento de mais de $50 \%$ em ambos os sexos e em todas as faixas etárias no período estudado. O enforcamento representa $76 \%$ dos casos entre os homens e $73 \%$ entre as mulheres.

Conclusões: $\mathrm{O}$ extremo oeste catarinense apresenta coeficientes de mortalidade por suicídio acima da média nacional e estadual, sendo comparável aos maiores do país. O perfil epidemiológico da região guarda semelhanças com o Rio Grande do Sul que devem ser investigadas em estudos seguintes.

Descritores: Suicídio, epidemiologia, Santa Catarina, Brasil.

\begin{abstract}
Introduction: The State of Santa Catarina is located in Southern Brazil. The suicide rate in this state is one of the largest in the country. Preliminary studies showed that the westernmost region of Santa Catarina has the largest rate in the state. Descriptive studies about suicide epidemiological profile in this area are not available.

Method: A descriptive study of suicide rate in the west of Santa Catarina was performed, stratified by gender and age from 1980 to 2005. Methods of suicide are also described. The data were collected from the Departamento de Informática do Sistema Único de Saúde (Information Technology Department of the Brazilian Unified Health System - DATASUS).

Results: Mean suicide rate was $10 \mathrm{cases} / 100,000$ inhabitants in western Santa Catarina. The male rate was three times higher than that of females. There was a $50 \%$ increase in suicide rates during the study period, independent of gender or age.

Hanging accounted for $76 \%$ of male and $73 \%$ of female deaths.

Conclusions: This study confirms that the westernmost region of Santa Catarina has suicide rates higher than the state and national levels, comparable to the highest rates in the country. The epidemiological profile has similarities with the State of Rio Grande do Sul and it should be investigated in further studies.

Keywords: Suicide, epidemiology, Santa Catarina, Brazil.
\end{abstract}




\section{Introdução}

Dados divulgados pela Organização Mundial de Saúde (OMS) indicam que as mortes por suicídio aumentaram $60 \%$ nos últimos 45 anos; o coeficiente de mortalidade mundial por suicídio no ano de 1995 foi de 16 casos/100.000 habitantes ${ }^{1}$. As estimativas da OMS apontam que cerca de 1 milhão de pessoas cometeram suicídio no ano 2000 , o que representaria uma morte a cada 40 segundos ${ }^{2}$.

A descrição do perfil epidemiológico do suicídio no Brasil vem crescendo significativamente nas últimas décadas. Em 2005, Mello-Santos et al. publicaram um estudo sobre as taxas nacionais de morte por suicídio entre os anos de 1980 e 2000. Os autores encontraram uma média de três a quatro suicídios/100.000 habitantes no Brasil, sendo a incidência quatro vezes maior entre homens e com taxas crescentes nas faixas etárias mais jovens ${ }^{3}$. No mesmo ano, o Ministério da Saúde concluiu um estudo que replicou esses achados e ainda revelou diferenças regionais nas taxas de suicídio no país. Os resultados revelaram que a Região Sul e mais especificamente o estado do Rio Grande do Sul (RS) detiveram os maiores coeficientes de mortalidade por suicídio no Brasil, chegando a 11/100.000 habitantes nesse estado ${ }^{4}$. As especificidades do suicídio no estado do RS já vêm sendo estudadas há mais de uma década pelo grupo "Agrotóxicos e outros fatores de risco para o suicídio no RS" ${ }^{\prime, 6}$. Esses mesmos pesquisadores publicaram um estudo detalhado sobre o perfil epidemiológico do suicídio no RS, indicando um aumento nos coeficientes de morte por suicídio entre 1980 e $1999^{6,7}$. Os estudos indicam ainda que, para cada suicídio consumado, ocorram pelo menos 10 tentativas sérias, que demandam atenção médica e aumento de custos para o sistema público ${ }^{2,3}$.

O estado de Santa Catarina (SC) é parte integrante da Região Sul do Brasil. Os estudos disponíveis indicam que o coeficiente de mortalidade por suicídio nesse estado gira em torno de 7/100.000 habitantes, o que representa quase o dobro da média nacional. Assim como no Brasil, as taxas de morte por suicídio em SC também apresentam variações regionais significativas. Um estudo divulgado pela Secretaria Estadual de Saúde apontou que a mortalidade por suicídio na região extremo oeste de SC apresentou coeficiente de 11/100.000 habitantes no ano de 2001, sendo superior ao coeficiente de mortes por homicídio na região ${ }^{8}$.

Para fins administrativos, a Secretaria Estadual de Saúde divide o território catarinense em oito macrorregiões de saúde: extremo oeste, meio-oeste, planalto serrano, sul, Florianópolis, Vale do Itajaí, planalto norte e nordeste ${ }^{9}$ (Figura 1). O extremo oeste possui uma população estimada em 2007 de 689.521 habitantes e tem na agroindústria o seu principal componente econômico ${ }^{10}$. A sua população é composta de remanescentes de caboclos (primeiros colonizadores da região), mas recebeu o principal fluxo imigratório após a década de 1920 com a chegada de colonos oriundos do RS, na sua maioria descendentes dos imigrantes italianos e alemães ${ }^{11}$.

No entanto, apesar dos altos índices de suicídio na região, poucos estudos têm sido conduzidos no sentido de descrever o fenômeno, seja no aspecto médico, epidemiológico ou social. Recentemente, a Universidade Comunitária Regional de Chapecó (UNOCHAPECÓ) criou um grupo de pesquisa em epidemiologia clínica que tem, entre outros objetivos, delinear o perfil epidemiológico tanto dos óbitos por suicídio como do comportamento suicida na região.

O presente trabalho busca traçar algumas características epidemiológicas das mortes por suicídio no extremo oeste de SC entre os anos de 1980 e 2005. Pretende-se, com isso, criar evidências que subsidiem pesquisas futuras na área, bem como sirvam de auxílio na construção de políticas públicas de enfrentamento do problema.

\section{Método}

Estudo descritivo das seguintes características epidemiológicas dos óbitos por suicídio no extremo oeste de SC entre os anos de 1980 e 2005: a) coeficiente bruto de mortalidade; b) coeficiente proporcional e padronizado para gênero; c) coeficiente proporcional e padronizado para faixa etária e; d) descrição dos meios empregados para o suicídio. Adicionalmente, calculouse o coeficiente de mortalidade por suicídio nas oito macrorregiões do estado para fins de comparação com os índices da região estudada. A região analisada (extremo oeste) foi definida conforme a divisão das macrorregiões de saúde da Secretaria Estadual de Saúde de Santa Catarina ${ }^{9}$, devidamente descriminada pelo Departamento de Informática do Sistema Único de Saúde (DATASUS) e pelo Instituto Brasileiro de Geografia e Estatística (IBGE).

Os dados utilizados foram extraídos do Sistema de Informação em Mortalidade (SIM), publicados pelo DATASUS, disponíveis no endereço eletrônico do Ministério da Saúde ${ }^{12}$. Os dados populacionais foram obtidos na página do IBGE na internet ${ }^{10}$.

Para o cálculo do número de suicídios entre os anos de 1980 e 1995, utilizou-se a nona revisão da Classificação Internacional de Doenças (CID-9), compreendendo as seguintes categorias:

- E950 - suicídio por sólidos ou líquidos;

- E951 - suicídio por gás doméstico;

- E952 - suicídio por outros gases ou vapores; 


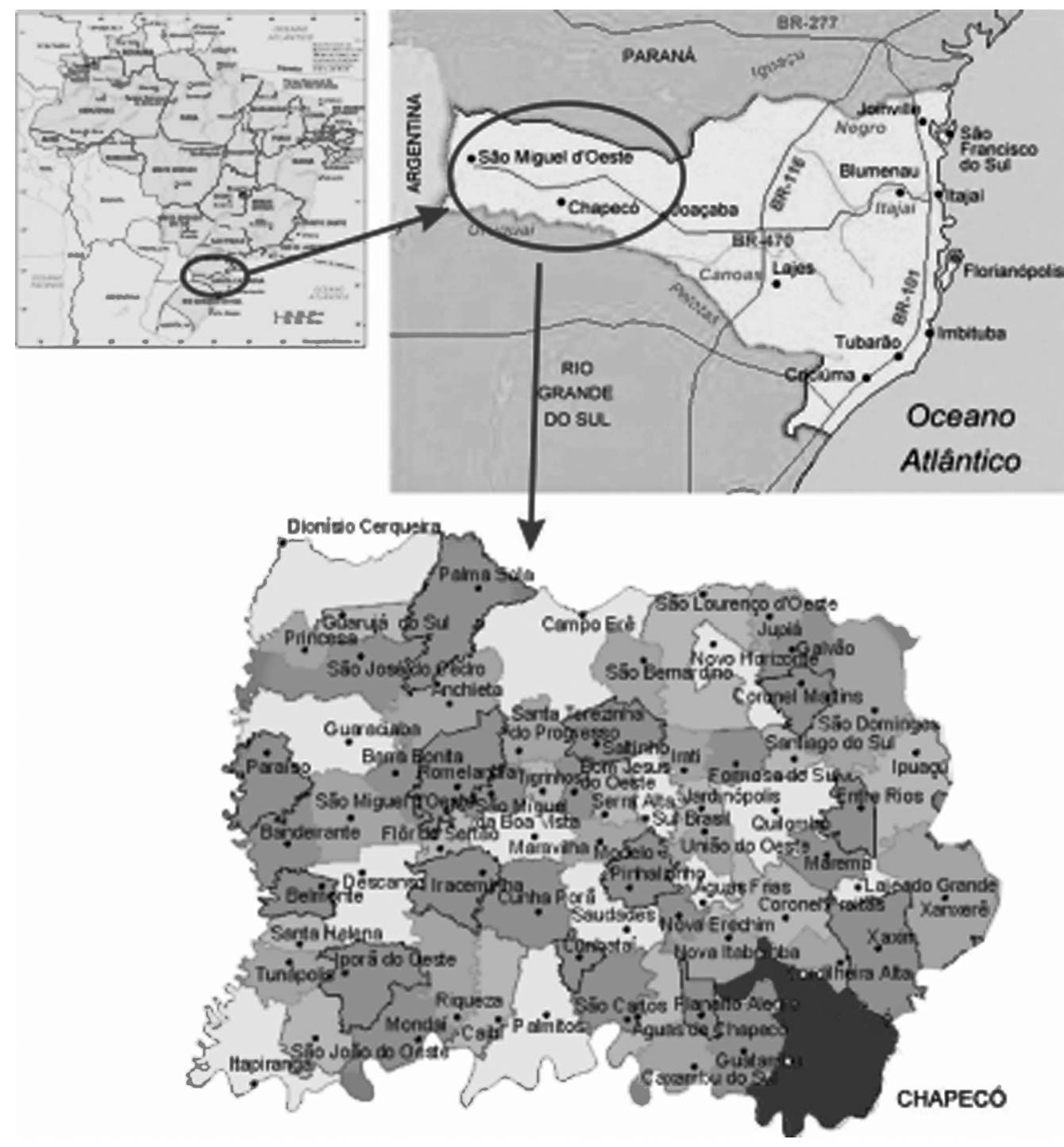

Figura 1 - Localização geográfica da região extremo oeste de Santa Catarina

- E953 - suicídio por enforcamento, estrangulamento ou sufocação;

- E954 - suicídio por submersão ou afogamento;

- E955 - suicídio por arma de fogo e explosivos;

- E956 - suicídio por instrumento cortante penetrante;

- E957 - suicídio por precipitação de lugar elevado;

- E958 - suicídio por outros procedimentos ou não especificado;

- E959 - efeitos tardios de lesões auto-infligidas.

Para o período entre 1996 e 2005, foi utilizada a décima revisão da Classificação Internacional de Doenças (CID-10), incluindo as categorias X60 a X84 (lesões autoprovocadas intencionalmente) e Y87.0 (seqüelas de lesões provocadas intencionalmente). A CID-10 descreve as lesões autoprovocadas intencionalmente da seguinte forma:

- X60: auto-intoxicação por exposição, intencional, a analgésicos, antipiréticos e antirreumáticos, não-opiáceos;

- X61: auto-intoxicação por exposição, intencional, a drogas anticonvulsivantes (antiepilépticos), sedativos, hipnóticos, antiparkinsonianos e psicotrópicos não classificados em outra parte;

- X62: auto-intoxicação por exposição, intencional, a narcóticos e psicodislépticos (alucinógenos) não classificados em outra parte; 
- X63: auto-intoxicação por exposição, intencional, a outras substâncias farmacológicas de ação sobre o sistema nervoso autônomo;

- X64: auto-intoxicação por exposição, intencional, a outras drogas, medicamentos e substâncias biológicas e às não-especificadas;

- X65: auto-intoxicação voluntária por álcool;

- X66: auto-intoxicação intencional por solventes orgânicos, hidrocarbonetos halogenados e seus vapores;

- X67: auto-intoxicação intencional por outros gases e vapores;

- X68: auto-intoxicação por exposição, intencional, a pesticidas;

- X69: auto-intoxicação por exposição, intencional, a outros produtos químicos e substâncias nocivas não especificadas;

- X70: lesão autoprovocada intencionalmente por enforcamento, estrangulamento e sufocação;

- X71: lesão autoprovocada intencionalmente por afogamento e submersão;

- X72: lesão autoprovocada intencionalmente por disparo de arma de fogo de mão;

- X73: lesão autoprovocada intencionalmente por disparo de espingarda, carabina ou arma de fogo de maior calibre;

- X74: lesão autoprovocada intencionalmente por disparo de outra arma de fogo e de arma de fogo não especificada;

- X75: lesão autoprovocada intencionalmente por dispositivos explosivos;

- X76: lesão autoprovocada intencionalmente pela fumaça, pelo fogo e por chamas;

- X77: lesão autoprovocada intencionalmente por vapor de água, gases ou objetos quentes;

- X78: lesão autoprovocada intencionalmente por objeto cortante ou penetrante;

- X79: lesão autoprovocada intencionalmente por objeto contundente;

- X80: lesão autoprovocada intencionalmente por precipitação de um lugar elevado;

- X81: lesão autoprovocada intencionalmente por precipitação ou permanência diante de um objeto em movimento;

- X82: lesão autoprovocada intencionalmente por impacto de um veículo a motor;

- X83: lesão autoprovocada intencionalmente por outros meios especificados;

- X84: lesão autoprovocada intencionalmente por meios não especificados.

Devido às diferenças entre a nona e a décima edição da CID, a categorização dos meios empregados para o suicídio foi organizada através do agrupamento das categorias similares. Dessa maneira, foram definidas como "enforcamento" as categorias E953 da CID-9 e X70 da CID-10; como "arma de fogo" as categorias E955 e X72-75; como "envenenamento" as categorias E950, E952 e X60-69; e como "outras" as demais.

Foram construídas séries históricas para o período de 1980 a 2005 . Esse período foi selecionado devido à disponibilidade de dados de mortalidade e população no DATASUS e IBGE. As faixas etárias foram descritas conforme a padronização da $\mathrm{OMS}^{13} \mathrm{e}$ agrupadas em quatro grandes grupos: a) 10 aos 19 anos; b) 20 aos 39 anos; c) 40 aos 59 anos; d) 60 anos ou mais. Os dados foram analisados através do programa TABWIN do Ministério da Saúde.

\section{Resultados}

Ocorreram 1.669 suicídios no extremo oeste catarinense entre 1980 e 2005 . Desse total, 11 casos tinham a idade ignorada, sendo 10 do sexo masculino e um do sexo feminino. Esses dados foram excluídos das análises conforme a faixa etária, mas foram mantidos no cálculo segundo o gênero.

No período analisado (1980 a 2005), o estado de SC apresentou um coeficiente médio de mortalidade por suicídio igual a sete casos/100.000 habitantes, enquanto a média brasileira não passou de quatro óbitos para cada 100.000 habitantes. O extremo oeste catarinense representou $20 \%$ do total de suicídios no estado nesses 25 anos (1.669 casos de um total de 8.248), ficando atrás somente da região do Vale do Itajaí, que totalizou $21 \%$ dos óbitos. O coeficiente médio de mortalidade do extremo oeste indicou um índice de 10 mortes/ 100.000 habitantes, sendo o maior do estado (Tabela 1). Todas as macrorregiões apresentaram um crescimento no número de suicídios no período estudado, praticamente dobrando os seus índices entre o ano de 1980 e 2005.

Os coeficientes no extremo oeste variaram de seis até 13 mortes/100.000 habitantes, mas desde o ano de 1997 vêm se mantendo entre 10 ou mais casos (Tabela 2). Os indivíduos do sexo masculino corresponderam a $78 \%$ do total de suicídios no período analisado. A razão entre os coeficientes padronizados por gênero indicam uma proporção média de 3:1 entre homens e mulheres, sendo o mesmo encontrado em estudos nacionais ${ }^{3,4}$. No entanto, tanto o número de óbitos quanto o coeficiente no sexo feminino cresceram nos últimos 25 anos e apresentaram uma média superior à população brasileira; o coeficiente médio de suicídios entre mulheres no extremo oeste foi de 4/100.000 entre 1980 e 2005. 
Tabela 1 - Coeficientes médios de mortalidade por suicídio nas macrorregiões de saúde de Santa Catarina entre 1980 e 2005

\begin{tabular}{lcc}
\hline Macrorregião & Coeficiente por 100 mil habitantes & $\mathbf{N}^{\mathbf{0}}$ óbitos \\
\hline Extremo oeste & 10 & 1.669 \\
Meio oeste & 08 & 1.087 \\
Vale do Itajaí & 07 & 1.744 \\
Planalto serrano & 06 & 462 \\
Planalto norte & 06 & 350 \\
Nordeste & 06 & 1.101 \\
Florianópolis & 05 & 892 \\
Sul & 05 & 943 \\
Total & 07 & 8.248 \\
\hline
\end{tabular}

Tabela 2 - Perfil epidemiológico do suicídio no extremo oeste segundo o gênero

\begin{tabular}{ccccccc}
\hline & \multicolumn{2}{c}{ Sexo masculino } & \multicolumn{2}{c}{ Sexo feminino } & \multicolumn{2}{c}{ Total } \\
\cline { 2 - 7 } Ano & Óbitos & Coef* & Óbitos & Coef* & Óbitos & Coef* \\
\hline 1980 & 31 & 10 & 5 & 2 & 36 & 6 \\
1981 & 30 & 10 & 9 & 3 & 39 & 6 \\
1982 & 35 & 11 & 11 & 4 & 46 & 8 \\
1983 & 42 & 13 & 20 & 7 & 62 & 10 \\
1984 & 44 & 14 & 13 & 4 & 57 & 9 \\
1985 & 28 & 9 & 7 & 2 & 35 & 6 \\
1986 & 30 & 9 & 7 & 2 & 37 & 6 \\
1987 & 33 & 10 & 7 & 2 & 40 & 6 \\
1988 & 48 & 15 & 15 & 5 & 63 & 10 \\
1989 & 51 & 16 & 13 & 4 & 64 & 10 \\
1990 & 54 & 16 & 18 & 6 & 72 & 11 \\
1991 & 56 & 17 & 20 & 6 & 76 & 12 \\
1992 & 53 & 16 & 17 & 5 & 70 & 11 \\
1993 & 55 & 16 & 7 & 2 & 62 & 9 \\
1994 & 55 & 16 & 13 & 4 & 68 & 10 \\
1995 & 47 & 13 & 15 & 4 & 62 & 9 \\
1996 & 49 & 15 & 13 & 4 & 62 & 9 \\
1997 & 67 & 20 & 17 & 5 & 84 & 12 \\
1998 & 70 & 20 & 16 & 5 & 86 & 13 \\
1999 & 66 & 19 & 15 & 4 & 81 & 12 \\
2000 & 67 & 20 & 15 & 4 & 82 & 12 \\
2001 & 62 & 18 & 18 & 5 & 80 & 12 \\
2002 & 63 & 18 & 22 & 7 & 85 & 13 \\
2003 & 58 & 17 & 19 & 6 & 77 & 11 \\
2004 & 52 & 15 & 19 & 6 & 71 & 10 \\
2005 & 54 & 16 & 18 & 5 & 72 & 11 \\
Total & 1.299 & 15 & 369 & 4 & 1.669 & 10 \\
\hline & & 9 & & & &
\end{tabular}

* Coeficiente/100.000 habitantes padronizado pela população.

$\mathrm{Na}$ análise estratificada por faixa etária, o coeficiente de mortes por suicídio apresentou índices progressivamente mais altos conforme o avanço da idade em todos os anos estudados e em ambos os sexos (Tabela 3). Por outro lado, em números absolutos, a maioria das mortes ocorreu na faixa entre
20 e 49 anos. Os coeficientes masculinos variaram de 4/100.000 na faixa entre 10 e 19 anos até 60/ 100.000 nos indivíduos acima de 60 anos de idade. Esses índices foram calculados considerando o número de suicídios e a população média do período para cada estrato etário. 
Tabela 3 - Mortalidade por suicídio conforme faixa etária e gênero

\begin{tabular}{|c|c|c|c|c|c|c|c|c|c|c|}
\hline \multirow[t]{2}{*}{ Sexo/idade } & \multicolumn{2}{|c|}{ 1980-1985 } & \multicolumn{2}{|c|}{ 1986-1990 } & \multicolumn{2}{|c|}{ 1991-1995 } & \multicolumn{2}{|c|}{ 1996-2000 } & \multicolumn{2}{|c|}{ 2001-2005 } \\
\hline & Óbitos & Coef* & Óbitos & Coef* & Óbitos & Coef* & Óbitos & Coef* & Óbitos & Coef* \\
\hline \multicolumn{11}{|l|}{ Masculino } \\
\hline 10-19 & 15 & 4 & 15 & 4 & 18 & 5 & 14 & 4 & 14 & 4 \\
\hline $20-39$ & 72 & 13 & 78 & 15 & 112 & 20 & 116 & 28 & 105 & 19 \\
\hline $40-59$ & 87 & 35 & 82 & 33 & 91 & 33 & 118 & 38 & 11 & 32 \\
\hline$>60$ & 31 & 37 & 39 & 47 & 44 & 46 & 69 & 60 & 59 & 46 \\
\hline \multicolumn{11}{|l|}{ Feminino } \\
\hline 10-19 & 15 & 4 & 6 & 1 & 11 & 3 & 9 & 3 & 7 & 1 \\
\hline $20-39$ & 29 & 5 & 24 & 5 & 29 & 5 & 26 & 5 & 38 & 7 \\
\hline $40-59$ & 12 & 5 & 16 & 6 & 24 & 10 & 25 & 8 & 35 & 10 \\
\hline$>60$ & 9 & 15 & 14 & 16 & 8 & 9 & 16 & 11 & 16 & 10 \\
\hline
\end{tabular}

* Coeficiente por 100.000 habitantes, padronizado por gênero e população média do período.

Na avaliação estratificada por faixa etária e gênero, observou-se uma tendência de estabilização dos coeficientes entre os 10 e 39 anos, assim como uma queda para as idades acima de 40 anos no sexo masculino nos últimos 5 anos do período (2000-2005) (Figura 2). Já no sexo feminino, detectou-se a tendência de crescimento das taxas de suicídio entre os 20 e os 59 anos de idade no mesmo período; o estrato entre os 40 e os 59 anos de idade apresenta os maiores coeficientes nos últimos anos da análise, sugerindo uma mudança no perfil das mulheres que se suicidam na região extremo oeste (Figura 2).

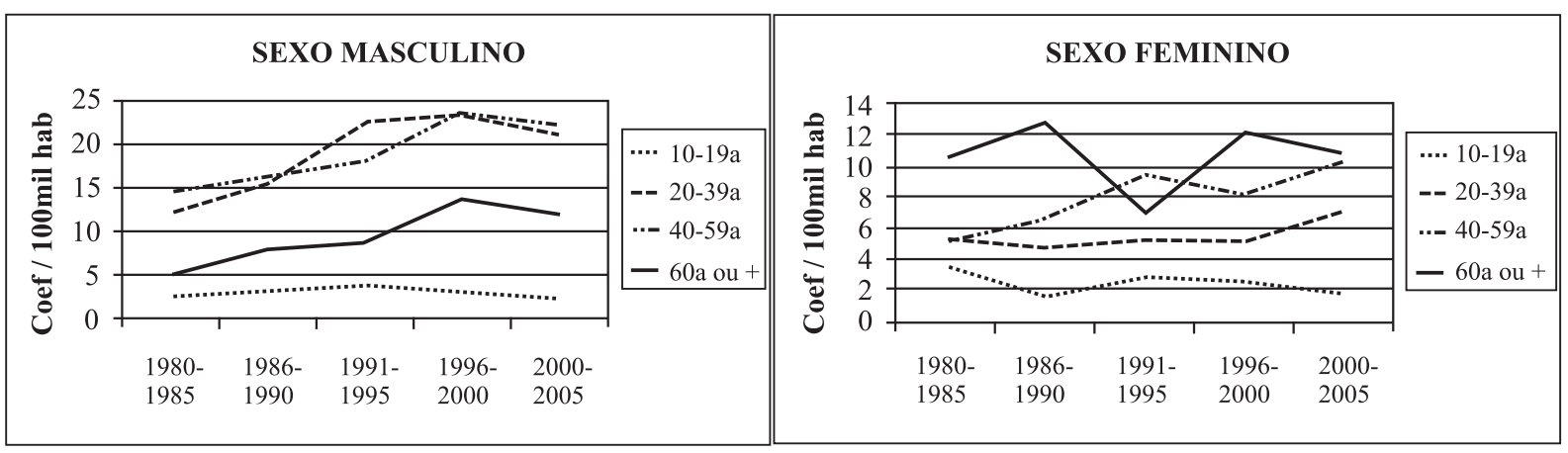

Figura 2 - Tendência dos suicídios conforme faixa etária e gênero (coeficiente padronizado por $n^{\circ}$ de óbitos/média da população sob risco no período x 100.000 habitantes)

O método mais empregado para o suicídio em ambos os sexos foi o enforcamento, responsável por mais de 70\% das mortes (categoria E953 na CID-9 e categoria X70 na CID-10) (Figura 3). O uso de armas de fogo respondeu por $13 \%$ das mortes no sexo masculino e $9 \%$ no feminino. O envenenamento, por sua vez, apresentou taxas de $5 \%$ entre os homens e $11 \%$ entre as mulheres. Diversos outros métodos estão registrados nas estatísticas do DATASUS, como afogamento e precipitação de lugar elevado, mas, isoladamente, cada um representava taxas em torno de $1 \%$, motivo pelo qual agrupamos essas categorias como "outros" (Figura 3).

\section{Discussão}

O estado de SC apresenta um dos maiores coeficientes nacionais de morte por suicídio (7/100.000). No entanto, a distribuição desses índices não é homogênea entre as diversas regiões do estado. O extremo oeste catarinense apresentou uma mortalidade média por suicídio de 10 casos para cada 100.000 habitantes entre os anos de 1980 e 2005. Estudos anteriores, com séries históricas mais restritas, já indicavam essa tendência ${ }^{8,14,15}$. Essa taxa foi a maior do estado. O estado de SC, apesar de possuir um território relativamente pequeno, se caracteriza por uma ampla 


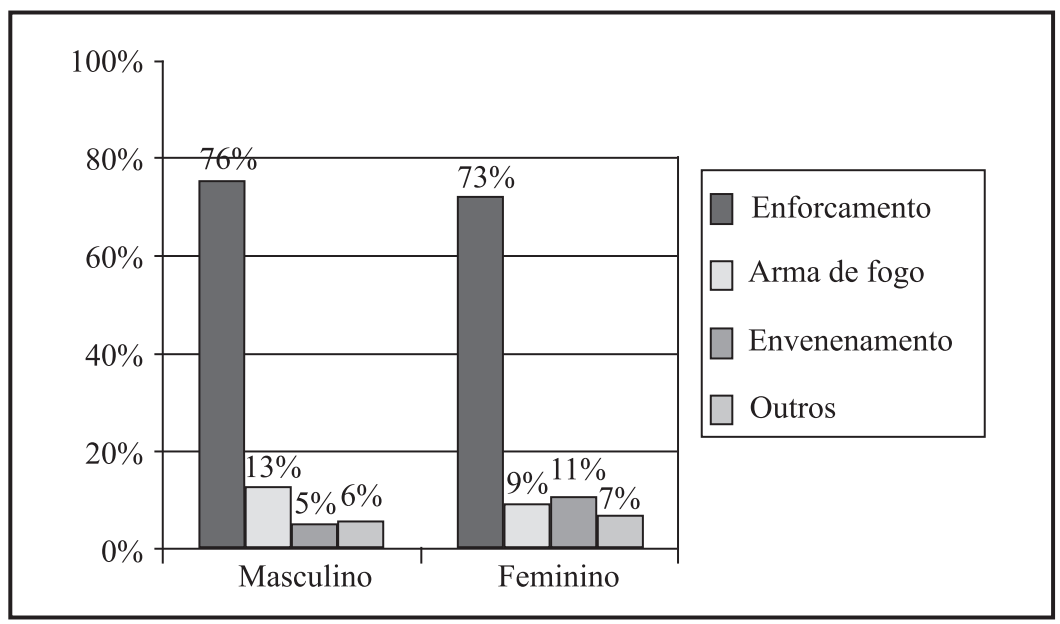

Figura 3 - Meios empregados para o suicídio no extremo oeste catarinense: estratificação por gênero no período de 1980 a 2005 (dados brutos e padronizados por gênero)

variação étnica e cultural entre as suas regiões ${ }^{11,16}$. O litoral catarinense, por exemplo, apresenta um processo histórico-político de colonização e desenvolvimento bem diverso do extremo oeste ${ }^{11,16}$. No que se refere ao suicídio, o extremo oeste detém taxas duas vezes maiores que as regiões de Florianópolis e Sul. Até que ponto essas diferenças étnicas e/ou culturais interferem nos coeficientes de suicídio ainda não sabemos, e um estudo descritivo como este não tem o poder de sugerir relações causais. No entanto, essa parece ser uma questão pertinente para estudos futuros.

Assim como no resto do país, tanto o número absoluto quanto o coeficiente de mortes por suicídio no extremo oeste cresceram nos últimos 25 anos. Desde o ano de 1997, o coeficiente anual tem se estabilizado entre 10 e 13 casos/100.000. Um estudo publicado pela OMS classificou a mortalidade por suicídio em quatro níveis, conforme o coeficiente apresentado $\left.{ }^{17}: 1\right)$ menor que 5/100.000 - baixo; 2) entre 5 e 15/100.000 - médio; 3) entre 15 e 30/100.000 - alto; e 4) acima de 30/100.000 - muito alto. Considerando tal classificação, os índices da região extremo oeste se encontram em nível médio, no mesmo patamar do RS. As explicações para a alta incidência nessa região ainda não estão claras. Uma comparação inicial com as evidências disponíveis indica que tanto o perfil étnico (descendentes de imigrantes europeus) quanto a atividade econômica (agroindustrial) estão presentes em áreas com altas taxas de suicídio no RS e SC. Outras variáveis, como escolaridade, nível socioeconômico e grau de investimento em saúde, também parecem estar correlacionadas com as taxas de suicídio ${ }^{7,18}$. O extremo oeste de $\mathrm{SC}$ vem apresentando um processo de urbanização nos últimos 10 anos, conforme dados do governo estadual. O Índice de Desenvolvimento Humano (IDH), que consiste em uma média de renda, educação e longevidade, está entre os níveis médio e alto, sendo um dos maiores do país ${ }^{16}$. O presente estudo é um levantamento preliminar do perfil epidemiológico do suicídio na região. A questão que se destaca é a necessidade de investigarmos a possível correlação entre esses fatores socioeconômicos e os índices de suicídio nessa região.

As taxas de suicídio por faixa etária e gênero vêm ao encontro das extensas descrições da literatura: incidência três a quatro vezes maior no sexo masculino e coeficientes crescentes conforme a idade. Quando analisada por estrato etário, a mortalidade no sexo feminino atinge coeficientes de 7/100.000 entre os adultos jovens e sobe para 10/100.000 na meia-idade e velhice, o que pode ser considerado médio pela classificação da OMS. Esses dados chamam a atenção porque indicam o agravamento do problema do suicídio em um grupo (mulheres) que tradicionalmente apresenta baixa incidência. Faria et al. ${ }^{7}$ apontaram uma correlação entre níveis crescentes de suicídio em trabalhadoras rurais do RS. É provável que o mesmo fenômeno se repita no extremo oeste de $\mathrm{SC}$, onde existe a predominância da agricultura familiar. Outro ponto de destaque é a tendência, indicada na Figura 2, de crescimento do suicídio entre mulheres adultas jovens e de meia-idade (entre 20 e 49 anos), enquanto as outras faixas etárias apresentam queda nos seus coeficientes. Entre os homens, observamos uma tendência de estabilização dos índices no último qüinqüênio analisado (2000-2005). Especificamente entre os adultos jovens (20-39 anos), a razão de suicídios homens/mulheres não chega a 3:1 entre os anos de 2000 e 2005. Portanto, o que se destaca aqui é a "progressão" de uma camada da população feminina em relação aos demais. Novamente, esses achados nos remetem a questionamentos 
posteriores: qual o perfil socioeconômico dessa população?

O método empregado para o suicídio parece ser o achado mais peculiar da região. Os enforcamentos foram responsáveis por $76 \%$ das mortes em homens e $73 \%$ em mulheres. O uso de armas de fogos foi de $13 \%$ entre os homens e $9 \%$ no sexo feminino. O envenenamento foi consideravelmente mais alto entre as mulheres $(11 \%$ contra 5\% no sexo masculino). No Brasil, o meio mais utilizado entre os homens, segundo estudo do Ministério da Saúde, é o uso de armas de fogo (44\%), e entre as mulheres, o enforcamento $(41 \%)^{4}$. Um estudo epidemiológico no RS apontou o enforcamento como responsável por $62 \%$ das mortes em geral ${ }^{6}$. Dessa maneira, vemos que no extremo oeste de SC o enforcamento é o método significativamente mais usado para o suicídio, enquanto que outras formas, como armas de fogo e envenenamento, apresentam participação discreta, quando comparada a estudos já publicados; no RS, as armas de fogo são responsáveis por mais de $20 \%$ dos suicídios ${ }^{6}$, enquanto a média brasileira se situa em $25 \%{ }^{19}$; o envenenamento apresentou taxas de $31 \%$ em estudo no município de Ribeirão Preto ${ }^{20}$. Este é outro ponto que ainda carece de estudos mais detalhados. A região do extremo oeste catarinense não pode ser considerada uma zona livre de armas de fogo; pelo contrário, é possível que o histórico de conflitos na região torne o acesso a essas armas ainda mais facilitado. No entanto, a utilização desse meio para o suicídio é baixa em comparação com outras regiões do Brasil. Da mesma forma, pela sua característica econômica, a utilização de pesticidas poderia elevar o número de envenenamentos, o que preliminarmente não parece ocorrer. Portanto, é provável que aspectos culturais e antropológicos estejam envolvidos na escolha do enforcamento como forma preferencial de suicídio. Leal $^{21}$ destaca que o enforcamento é um fenômeno intimamente ligado à cultura gaúcha. Segundo a autora, o suicídio pode ser visto como uma saída honrosa para o homem gaúcho que de alguma forma teve a sua honra ferida, e o enforcamento seria uma forma "masculina" de morrer. A colonização do extremo oeste catarinense foi fortemente influenciada pela cultura rio-grandense, uma vez que a maioria dos colonos que ocuparam o território a partir do século XX era oriunda do $\mathrm{RS}^{11}$. Uma especulação possível é que RS e extremo oeste catarinense sejam separados do ponto de vista políticoadministrativo, mas formem um cluster culturalmente homogêneo que teria alguma repercussão no perfil epidemiológico dos suicídios. A explicação antropológica, no entanto, não esclareceria por que o enforcamento é também o método preferencial entre as mulheres do extremo oeste catarinense.

A principal limitação do presente trabalho se refere a sua característica descritiva. Um estudo dessa natureza auxilia no diagnóstico de saúde e sistematização dos dados, mas prescinde da capacidade de estabelecer associações e estimar riscos. O presente trabalho tratase de um breve relatório das estatísticas disponíveis no DATASUS. Embora importante como "mapeamento" geral do extremo oeste, a ausência de análises mais detalhadas limita a capacidade de comparação e detecção de diferenças estatísticas. Alguns autores apontam que os estudos epidemiológicos sobre o suicídio no Brasil ainda carecem de métodos estatísticos refinados e sugerem a adoção de análises econométricas como forma de aperfeiçoamento dos trabalhos ${ }^{18}$. Outro ponto limitante é a possível subnotificação dos casos de suicídio no Brasil. Os dados de mortalidade baseiamse nos atestados de óbito, e sua qualidade depende muito da interpretação subjetiva do profissional que o preenche. Meneghel ${ }^{6}$ afirma que os suicídios podem ser notificados como causa externa ignorada devido ao tabu que cerca esse tipo de morte.

\section{Conclusão}

Diversas pesquisas já publicadas indicam que SC apresenta um dos maiores coeficientes nacionais de morte por suicídio. Os achados desse estudo confirmaram pesquisas anteriores que apontavam a região extremo oeste de $\mathrm{SC}$ como a de maior mortalidade por suicídio no estado. O presente estudo tem como característica adicional o fato de contar com uma série histórica extensa (1980-2005), o que ainda não havia ocorrido nas estatísticas descritivas sobre o suicídio em SC.

As características epidemiológicas dessa região assemelham-se às do estado do RS, com um alto coeficiente bruto (média de 10/100.000 habitantes) e o enforcamento como método utilizado na maioria absoluta dos casos. Assim como na maioria dos locais no mundo, a incidência de suicídios é cerca de três vezes maior entre os homens e apresenta coeficientes crescentes conforme a faixa etária.

Nos últimos 5 anos da série estudada, os índices de suicídio, embora altos, têm apresentado tendência a estabilização ou mesmo queda. As razões desse achado devem ser investigadas posteriormente, mas alguns estudos sugerem que a disseminação dos tratamentos psiquiátricos possa estar relacionada com essas reduções ${ }^{1}$. No entanto, entre as mulheres dos 20 aos 49 anos, as taxas de suicídio vêm aumentando nesse mesmo período.

Esses resultados podem auxiliar na elaboração de novas pesquisas a respeito do fenômeno na região. Várias questões surgem a partir dos achados deste trabalho. Entre elas, destacamos: qual o perfil socioeconômico do suicídio? Existiria uma zona 
endêmica de suicídios entre o RS e o extremo oeste de $\mathrm{SC}$, já que o perfil epidemiológico e aspectos culturais são semelhantes entre as populações? Por que o enforcamento é o método utilizado em mais de $70 \%$ dos casos?

Os resultados indicam que o suicídio é um relevante problema de saúde pública nessa região de SC. Dados de outras pesquisas indicam que somente os acidentes de trânsito apresentam índices superiores aos do suicídio na mortalidade por causas externas no extremo oeste de $\mathrm{SC}^{8}$. A colaboração entre pesquisadores de diversas áreas, como medicina, ciências sociais, antropologia e economia, pode ser útil na construção de conhecimentos cientificamente fundamentados sobre o suicídio no extremo oeste de SC.

\section{Referências}

1. Bahls SC, Botega NJ. Epidemiologia das tentativas de suicídio e dos suicídios. In: Mello MF, Mello AF, Kohn R ed. Epidemiologia da saúde mental no Brasil. Porto Alegre: Artmed;2007. p.151-71.

2. World Health Organization. Multisite intervetion study on suicidal behaviours. Geneva, 2002. Disponível em: http://www.who.int/ mental_health/media/en/254.pdf (acessado em 20/01/08).

3. Mello-Santos C, Wang YP, Bertolote JM. Epidemiology of suicide in Brazil (1980-2000): characterization of age and gender rates of suicide. Revista Brasileira de Psiquiatria. 2005;27(2):131-4.

4. D`Oliveira CFA. Perfil epidemiológico dos suicídios. Brasil e regiões, 1996 a 2002. Ministério da Saúde, 2005. Disponível em: http://portal.saude.gov.br/portal/arquivos/pdf/Suicidios.pdf (Acessado em 20/01/08).

5. Csillag C. Brazil's soaring suicide rate revealed. Lancet. 1996;348:1651

6. Meneghel SN, Victora CG, Faria NMX, Carvalho LA, Falk JW. Características epidemiológicas do suicídio no Rio Grande do Sul. Rev Saude Publica. 2004;38(6):804-10.

7. Faria NMX, Victora CG, Meneghel SN, Carvalho LA, Falk JW. Suicide rates in the state of Rio Grande do Sul, Brazil: association with socioeconomic, cultural and agricultural factors. Caderno Saude Publica. 2006;22(12):2611-21.
8. Peixoto HCG. Redução da morbimortalidade por acidentes e violências: diagnóstico do problema em Santa Catarina. Secretaria Estadual de Saúde de Santa Catarina, 2003. Disponível em: www.saude.sc.gov.br/gestores/sala_de_leitura/doc_tecnicos (Acessado em 20/01/08).

9. Secretaria Estadual de Saúde de Santa Catarina. Política de Saúde Descentralizada. Secretaria Estadual de Saúde, 2008. Disponível em: www.saude.sc.gov.br (Acessado em 20/01/08).

10. Instituto Brasileiro de Geografia e Estatística. Banco de dados por estado. IBGE, 2008. Disponível em: www.ibge.gov.br (Acessado em 20/01/08).

11. Woloszyn N. Em busca da terra: Colonização e exploração de madeiras no Oeste Catarinense. Universidade do Contestado, 2008. Disponível em: http://www.pesquisa.uncnet.br/pdf/historia/ busca_terra_colonizacao_exploracao_madeiras_oeste_catarinense.pdf (Acessado em 20/01/08).

12. Brasil, Ministério da Saúde. Departamento de Informática do SUS. Disponível em: www.datasus.gov.br (Acessado em 20/01/08).

13. Ahmad OB, Boschi-Pinto C, Lopez AD, Murray CJL, Lozano R, Inoue M. Age standartization of rates: a new who standard. World Health Organization -GPE discussion papers series. 2000;31:1-14.

14. Peixoto HCG. Análise da mortalidade em Santa Catarina, 2003. Secretaria Estadual de Saúde de Santa Catarina, 2003. Disponível em: http://www.saude.sc.gov.br/gestores/sala_de_leitura (Acessado em 20/01/08)

15. Peixoto HCG, Souza ML. O indicador anos potenciais de vida perdidos e a ordenação das causas de morte em Santa Catarina 1995. Inf Epidemiol SUS. 1999;8(1):17-25.

16. Governo do Estado de Santa Catarina. Regiões de Santa Catarina. Disponível em: www.sc.gov.br(Acessado em 20/01/08).

17. Diekstra RF, Gulbinat W. The epidemiology of suicidal behaviour:a review of three continents. World Health Stat Q. 2003;46(1):52-68.

18. Shikida C, Vilhena RA, Araujo Junior AFA. Teoria econômica do suicídio: estudo empírico para o Brasil. Centro de Economia Aplicada e Estratégia Empresarial-IBMEC/MG,2006. Disponível em: http://www.ceaee.ibmecmg.br/working.htm (Acessado em 20/ 01/08).

19. Santos SM, Barcellos C, Carvalho MS, Flores R. Detecção de aglomerados espaciais de óbitos por causa violentas em Porto Alegre, Rio Grande do Sul, Brasil, 1996. Cadernos de Saude Publica. 2001;17(5):1141-51.

20. Marin-León L, Barros MBA. Mortes por suicídio: diferenças de gênero e nível socioeconômico. Rev Saude Publica. 2003;37(3):357-63.

21. Leal OF. Suicídio, honra e masculinidade na cultura gaúcha. Cad Antropologia UFRGS. 1992;07-14. 